\title{
Randomized phase II study of the PDGFRa antibody olaratumab plus liposomal doxorubicin versus liposomal doxorubicin alone in patients with platinum-refractory or platinum-resistant advanced ovarian cancer
}

William P. McGuire ${ }^{1 *}$, Richard T. Penson², Martin Gore ${ }^{3}$, Antonio Casado Herraez ${ }^{4}$, Patrick Peterson ${ }^{5}$, Ashwin Shahir ${ }^{6}$ and Robert Ilaria Jr, $r^{5,7}$

\begin{abstract}
Background: Olaratumab is a platelet-derived growth factor receptor-a (PDGFRa)-targeting monoclonal antibody blocking PDGFRa signaling. PDGFRa expression is associated with a more aggressive phenotype and poor ovarian cancer outcomes. This randomized, open label phase II study evaluated olaratumab plus liposomal doxorubicin compared with liposomal doxorubicin alone in advanced ovarian cancer patients.

Methods: Patients with platinum-refractory or platinum-resistant advanced ovarian cancer were randomized 1:1 to receive liposomal doxorubicin ( $40 \mathrm{mg} / \mathrm{m}^{2}$, intravenous infusion) administered every 4 weeks with or without olaratumab (20 mg/kg, IV infusion) every 2 weeks. Patients were stratified based on prior response to platinum therapy (refractory vs resistant). The primary efficacy endpoint was progression-free survival (PFS). Secondary endpoints included overall survival (OS), objective response rate, duration of response, and safety.
\end{abstract}

Results: A total of 123 patients were treated (62 olaratumab+liposomal doxorubicin; 61 liposomal doxorubicin). Median PFS was 4.2 months for olaratumab+liposomal doxorubicin and 4.0 months for liposomal doxorubicin (stratified hazard ratio $[H R]=1.043 ; 95 \%$ confidence interval $[\mathrm{Cl}]$ 0.698-1.558; $p=0.837$ ). Median OS was 16.6 months and 16.2 months in the olaratumab+liposomal doxorubicin and liposomal doxorubicin arms, respectively $(H R=1.098 ; 95 \% \mathrm{Cl} 0.71-1.71)$. In the platinum-refractory subgroup, median PFS was 5.5 months ( $95 \% \mathrm{Cl} 1.6-9.2)$ and 3.7 months $(95 \% \mathrm{Cl} 1.9-9.2)$ in the olaratumab+liposomal doxorubicin $(n=15)$ and liposomal doxorubicin arms $(n=16)$, respectively $(H R=0.85 ; 95 \% \mathrm{Cl} 0.38-$ 1.91). Overall, $59.7 \%$ (olaratumab+liposomal doxorubicin) and $65.6 \%$ (liposomal doxorubicin) of patients reported grade $\geq$ 3 adverse events regardless of causality. The most common treatment-emergent adverse events (all grades) regardless of causality were fatigue related (61\%), nausea (57\%), and constipation (52\%) with olaratumab+liposomal doxorubicin and nausea (64\%), fatigue related (62\%), and mucositis (46\%) with liposomal doxorubicin.

Conclusions: The addition of olaratumab to liposomal doxorubicin did not result in significant prolongation of PFS or OS in platinum-resistant or platinum-refractory ovarian cancer.

(Continued on next page)

\footnotetext{
* Correspondence: william.mcguire@vcuhealth.org

${ }^{1}$ Virginia Commonwealth University, 1201 E Marshall St, Room 11-210,

Richmond, VA 23298, USA

Full list of author information is available at the end of the article
}

(c) The Author(s). 2018 Open Access This article is distributed under the terms of the Creative Commons Attribution 4.0 International License (http://creativecommons.org/licenses/by/4.0/), which permits unrestricted use, distribution, and reproduction in any medium, provided you give appropriate credit to the original author(s) and the source, provide a link to the Creative Commons license, and indicate if changes were made. The Creative Commons Public Domain Dedication waiver (http://creativecommons.org/publicdomain/zero/1.0/) applies to the data made available in this article, unless otherwise stated. 
(Continued from previous page)

Trial registration: ClinicalTrials.gov identifier: NCT00913835; registered June 2, 2009.

Keywords: Ovarian cancer, Olaratumab, Liposomal doxorubicin, Platinum refractory, Platinum resistant

\section{Background}

Ovarian cancer is a family of many diseases, each with specific histology, risk factors, molecular characteristics, and treatment [1]. Epithelial ovarian cancer (EOC) comprises 90\% of cases; of these, serous is the most common subtype [1]. The current standard treatment for EOC of all subtypes involves debulking surgery followed by combination chemotherapy with a platinum plus taxane base [2-4]. Patients who relapse after first-line treatment may be classified into 1 of 2 subgroups: those with platinum-refractory/ -resistant disease and those with platinum-sensitive disease [5]. Although many agents are available for patients with platinum-resistant or -refractory disease who have also received first-line paclitaxel, there is still no definitive second-line treatment for these patients [2-4].

Several phase II clinical studies of patients with platinum-resistant or -refractory disease have demonstrated the benefit of using doxorubicin in combination therapy with other agents [6-12]. Liposomal doxorubicin is approved by the United States Food and Drug Administration and the European Medicine Agency for ovarian cancer in women who have failed platinum-based chemotherapy [13, 14]. Treatment guidelines recognize that combining traditional chemotherapeutic agents with drugs targeting growth factors/receptors may be more effective for treating platinum-resistant/-refractory recurrent ovarian cancer than chemotherapy alone $[3,4]$.

The platelet-derived growth factor receptors (PDGFRs: PDGFR $\alpha$ and PDGFR $\beta$ ) are transmembrane receptor tyrosine kinases that are activated by their cognate ligands [15]. Platelet-derived growth factor (PDGF)-AA binds PDGFR $\alpha$, whereas PDGF-AB and PDGF-BB recognize both PDGFR $\alpha$ and PDGFR $\beta$ [15]. Upon binding of circulating PDGF ligand, PDGFR $\alpha$ and $\beta$ subunits homodimerize or heterodimerize, undergo autophosphorylation, and activate downstream signal transduction molecules, including phosphoinositide 3-kinase, Ras, phospholipase $C \gamma$, and $\operatorname{Src}[16,17]$. PDGFR signaling plays a significant part in mesenchymal biology, including mesenchymal stem cell differentiation, proliferation, and angiogenesis $[18,19]$. Aberrant PDGF/PDGFR signaling is involved in the development and maintenance of cancer, and has been implicated in modulating the tumor or stromal microenvironment thus facilitating metastasis in several malignancies [16, 17]. The PDGF/ PDGFR axis has pro-angiogenic activity and may contribute to resistance to anti-vascular endothelial factor therapy [20].
Expression of PDGFR $\alpha$ has been reported in ovarian cancers, although the prevalence varies [21-23]. This may reflect the variety of methods and reagents used to measure PDGFR $\alpha$, with recent reports suggesting that some of the reagents used in previous studies may be nonspecific for PDGFR $\alpha$ [24]. A study by Matsuo et al. [25] on the extent of PDGFR $\alpha$ protein expression assessed in 176 human ovarian tumors revealed that PDGFR $\alpha$ expression was significantly associated with serous histology (serous vs nonserous, $77 \%$ vs $46 \%$, respectively; odds ratio, 4.0 ) and advanced stage (odds ratio, 1.7). The most common type of histology was high-grade serous ovarian carcinomas [25]. Among patients with high-grade serous tumors, PDGFR $\alpha$-expressing tumors were associated with significantly poorer survival outcomes (median OS, 51 months) compared to patients with PDGFR $\alpha$-nonexpressing tumors (median OS, 174 months; $p=0.014$ ) [25]. In addition, when controlled for age and stage, PDGFR $\alpha$ expression remained a significant variable for OS [25].

When present, PDGFR $\alpha$ may be stimulated in an autocrine loop by ovarian tumors co-expressing PDGF-AB [23]. This activation induced Akt- and mitogen-activated protein kinase (MAPK) -mediated proliferation of tumor cells [23]. In a clinical trial of patients who were platinum-resistant or -refractory, the PDGFR kinase inhibitor, imatinib, in combination with docetaxel showed an objective response rate (ORR) of $22 \%$ (5 of 23 patients) [26].

Olaratumab (LY3012207; formerly IMC-3G3) is a recombinant fully human immunoglobulin $G$ subclass 1 (IgG1) monoclonal antibody that specifically binds to PDGFR $\alpha$, blocking signaling of PDGF ligands [27]. The antibody inhibits PDGFR ligand-induced receptor autophosphorylation and phosphorylation of downstream signal transduction via Akt and MAPK [27]. Olaratumab has antitumor activity in in vivo tumor models thought to be driven by a PDGF-PDGFR $\alpha$ autocrine loop [27]. In mouse models of pediatric osteosarcoma and malignant rhabdoid tumor, olaratumab delayed tumor growth, and this activity was enhanced by chemotherapy (cisplatin or doxorubicin) [28]. Likewise, olaratumab alone and in combination with docetaxel significantly reduced tumor weight in in vivo xenograft models of ovarian carcinoma compared to control and docetaxel alone, respectively [25]. In a phase Ib/IIa study, the combination of olaratumab+doxorubicin significantly improved both progression-free survival (PFS; 6.6 vs 4.1 months in phase II) and overall survival (OS; 26.5 vs 14.7 months in phase II) relative to doxorubicin 
alone in patients with advanced soft tissue sarcoma [29]. The present phase II study was performed to evaluate the combination of olaratumab+liposomal doxorubicin vs liposomal doxorubicin alone in patients with platinum-refractory or platinum-resistant advanced ovarian cancer.

\section{Methods}

\section{Study design and patient enrollment}

This was a randomized, open-label, multicenter phase II study. The primary endpoint was PFS. Secondary endpoints included ORR, OS, duration of response, and safety. This study (NCT00913835) was conducted according to the Declaration of Helsinki and with approval from Institutional Review Boards of all participating study sites. All participants provided written informed consent prior to any study-related procedures. This manuscript adheres to CONSORT reporting guidelines.

\section{Study participants}

This study enrolled adult females ( $\geq 18$ years) with histologically or cytologically confirmed EOC, primary peritoneal carcinoma or fallopian tube cancer that was platinum-resistant or platinum-refractory. Patients must have completed 1 to 3 platinum-containing regimens for their disease. Platinum-refractory was defined as progression or persistent disease while receiving platinum-containing therapy; platinum-resistant was defined as disease recurrence $\leq 12$ months following platinum-containing chemotherapy.

Additional enrollment criteria included measurable disease according to the Response Evaluation Criteria in Solid Tumors (RECIST v1.0) guidelines and an Eastern Cooperative Oncology Group performance status (ECOG PS) score of $\leq 1$. Prior to enrollment, patients had to recover to grade $\leq 1$ from the effects of prior therapies according to National Cancer Institute Common Terminology Criteria for Adverse Events, Version 3.0 (NCI-CTCAE v3.0), with the exception of peripheral neuropathy (which must resolve

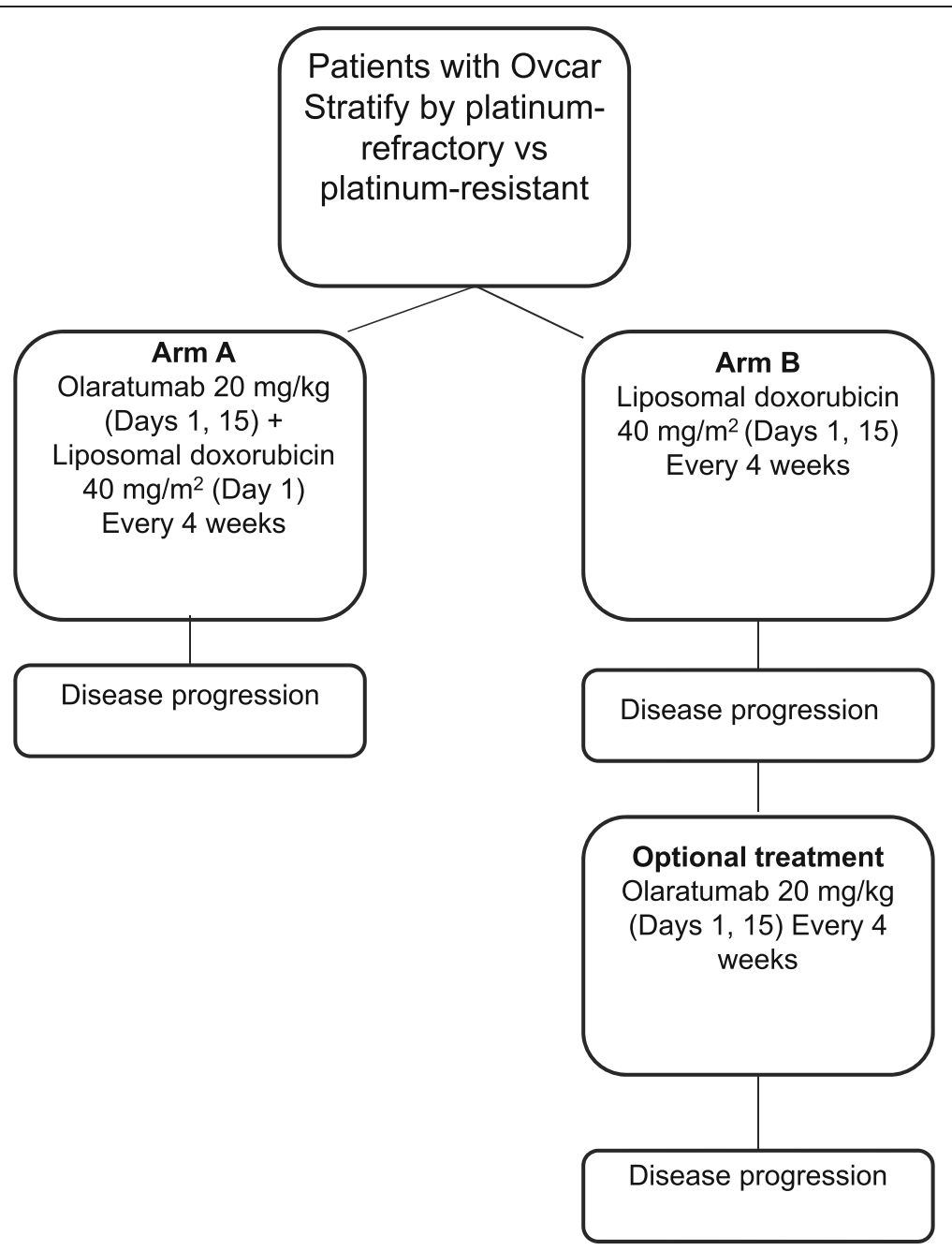

Fig. 1 Study design: open-label, nonblinded, multicenter, Phase II trial 
to grade $\leq 2)$. Patients with brain metastases, leptomeningeal disease, or increased level of cancer antigen 125 (CA125) in the absence of concomitant clinical or radiographic progression were excluded.

\section{Study procedures}

Study site personnel randomized patients using either a call-in Interactive Voice Response System (IVRS) or Interactive Web Response System (IWRS). The IVRS/ IWRS assigned a unique identification number to each patient. Patients were stratified based on the previous response to platinum therapy (refractory vs resistant). Within each stratum, patients were randomly assigned (1:1) to olaratumab+liposomal doxorubicin or liposomal doxorubicin alone. Patients in the olaratumab+liposomal doxorubicin arm received IV olaratumab at $20 \mathrm{mg} / \mathrm{kg}$ on days 1 and 15 and liposomal doxorubicin at $40 \mathrm{mg} / \mathrm{m}^{2}$ on day 1 of a 28-daycycle; patients in the liposomal doxorubicin arm received $40 \mathrm{mg} / \mathrm{m}^{2}$ on day 1 every 4 weeks (Fig. 1). Upon disease progression, patients in the liposomal doxorubicin arm could elect to receive olaratumab monotherapy (20 mg/kg every 2 weeks); data from this group are not presented due to small sample size.

Patients underwent radiographic disease assessment approximately every 8 weeks. Treatment was continued until disease progression, unacceptable toxicity, protocol noncompliance, or consent withdrawal. After the last study visit, follow-up information on additional anticancer treatment, disease progression, and survival was collected every 2 months for up to 2 years.

A safety review by the Safety Review Committee (SRC) was mandated after 6 patients were treated for at least 8 weeks on the liposomal doxorubicin+olaratumab arm. Any patient who discontinued for toxicity prior to 8 weeks was also included in the review. If the incidence of serious adverse events (SAE) markedly exceeded that expected of liposomal doxorubicin monotherapy, protocol modification, termination, or ongoing monitoring was considered by the SRC. There were no interim efficacy analysis performed for this study.

Table 1 Baseline demographics

Olaratumab + Liposomal Doxorubicin $(n=62)$ Liposomal Doxorubicin $(n=61)$

Total $(N=123)$

Sex, No. (\%)

Female

$62(100.0)$

$61(100.0)$

$123(100.0)$

Race, No. (\%)

$\begin{array}{ll}\text { Asian } & 1(1.6) \\ \text { Black or African American } & 5(8.1) \\ \text { Native Hawaiian or other Pacific Islander } & 0 \\ \text { White } & 54(87.1) \\ \text { Other } & 2(3.2) \\ \text { Ethnicity, No. (\%) } & \\ \text { Hispanic or Latino } & 1(1.6) \\ \text { Non-Hispanic or Latino } & 61(98.4) \\ \text { Missing } & 0\end{array}$

$3(4.9)$

4 (3.3)

1 (1.6)

$6(4.9)$

1 (1.6)

$1(0.8)$

54 (88.5)

108 (87.8)

ge, yrs

Mean (SD)

ECOG PS, No. (\%)

\begin{tabular}{|c|c|c|c|}
\hline 0 & $37(59.7)$ & $31(50.8)$ & $68(55.3)$ \\
\hline 1 & $25(40.3)$ & $30(49.2)$ & $55(44.7)$ \\
\hline Prior chemotherapy, No. (\%) & $62(100.0)$ & $61(100.0)$ & $123(100.0)$ \\
\hline \multicolumn{4}{|c|}{ Stratification factor (CRF), No. (\%) } \\
\hline Platinum-refractory & $13(21.0)$ & $17(27.9)$ & $30(24.4)$ \\
\hline Platinum-resistant & $49(79.0)$ & $44(72.1)$ & $93(75.6)$ \\
\hline \multicolumn{4}{|c|}{ Stratification factor (IVRS), No. (\%) } \\
\hline Platinum-refractory & $15(24.2)$ & $16(26.2)$ & $31(25.2)$ \\
\hline Platinum-resistant & $47(75.8)$ & $45(73.8)$ & $92(74.8)$ \\
\hline
\end{tabular}




\section{Statistical analyses}

A total of 110 evaluable patients were planned with 55 patients for each treatment arm. This sample size would provide a $69.3 \%$ power to detect an expected increase of median PFS from 12 weeks (liposomal doxorubicin monotherapy) to 18.5 weeks when liposomal doxorubicin was combined with olaratumab. Final analysis was planned to be performed when at least 99 PFS events were observed or all patients discontinued from study therapy. Additional assumptions included an accrual time of 55 weeks at a rate of 2 patients per week, a follow-up time of 28 weeks, and an $\alpha$-level of $10 \%$ using a 2 -sided test.

Efficacy data for the primary endpoint, which was PFS, and the secondary endpoints were analyzed in the modified intent-to-treat (mITT) population, which included all patients who were randomized and received any quantity of study drug; patients were categorized by the treatment arm to which they were randomized, regardless of the actual treatment received. For the primary endpoint, PFS, the Kaplan-Meier method was used to estimate the median PFS time, together with a 95\% confidence interval (CI). Comparison between arms was performed using the stratified log rank test, and hazard ratios (HRs) were determined by a Cox proportional hazards regression model. The log rank test and Cox regression models were used to compare the survival curves adjusting for prior response to platinum treatment as a stratification factor (platinum-refractory vs platinum-resistant). Sensitivity analyses for PFS using various censoring rules were defined in the separate statistical analysis plan. Additional analysis of PFS was performed for treatment effects across different subgroups. The subgroups were defined by the stratification factor (platinum-refractory, platinum-resistant), age ( $\leq 62$ years, $>62$ years), ECOG PS $(0,>0)$, progressive disease $(\mathrm{PD})$ in previous anticancer therapy (yes, no), duration of disease ( $\leq 15.21$ months, $>15.21$ months), and serum levels of CA125 $(\leq 64.3 \mathrm{kU} / \mathrm{L},>64.3 \mathrm{kU} / \mathrm{L})$.

Safety analysis was conducted on all patients who received any quantity of olaratumab, regardless of study eligibility (safety population). Patients were categorized by the treatment actually received, regardless of the arm to which they were randomized. An adverse event $(\mathrm{AE})$ was regarded as treatment-emergent if its onset date occurred any time after the administration of the first dose of study drug and up to 30 days after the last dose of study treatment (or up to any time if related to study treatment), or if it occurred prior to first dose date and worsened while on therapy.

Table 2 Patient disposition

\begin{tabular}{|c|c|c|c|}
\hline & \multicolumn{3}{|l|}{ No. (\%) of Patients } \\
\hline & Olaratumab + Liposomal Doxorubicin & Liposomal Doxorubicin & Total \\
\hline mITT population & 62 & 61 & 123 \\
\hline Treated & $62(100.0)$ & $61(100.0)$ & $123(100.0)$ \\
\hline On treatment ${ }^{a}$ & $1(1.6)$ & 0 & $1(0.8)$ \\
\hline Off treatment & $61(98.4)$ & $61(100.0)$ & $122(99.2)$ \\
\hline \multicolumn{4}{|c|}{ Reasons for discontinuation of study therapy } \\
\hline Adverse event & $2(3.2)$ & $7(11.5)$ & $9(7.3)$ \\
\hline Death & $2(3.2)$ & 0 & $2(1.6)$ \\
\hline PD per RECIST & $42(67.7)$ & $12(19.7)$ & $54(43.9)$ \\
\hline PD, symptomatic deterioration & $10(16.1)$ & $8(13.1)$ & $18(14.6)$ \\
\hline Withdrawal by patient & $1(1.6)$ & $3(4.9)$ & $4(3.3)$ \\
\hline Lost to follow-up & $1(1.6)$ & 0 & $1(0.8)$ \\
\hline Other & $3(4.8)$ & $3(4.9)$ & $6(4.9)$ \\
\hline \multicolumn{4}{|c|}{ Reasons for discontinuation for patients electing to receive olaratumab monotherapy after progression on liposomal doxorubicin } \\
\hline PD per RECIST & 0 & $26(42.6)$ & $26(21.1)$ \\
\hline PD, symptomatic deterioration & 0 & $2(3.3)$ & $2(1.6)$ \\
\hline On study ${ }^{a}$ & $1(1.6)$ & $1(1.6)$ & $2(1.6)$ \\
\hline Off study & 61 (98.4) & 60 (98.4) & $121(98.4)$ \\
\hline
\end{tabular}

$\mathrm{mIT}$, modified intent-to-treat; PD, progressive disease; RECIST, Response Evaluation Criteria in Solid Tumors. ${ }^{\text {Refers }}$ to those who were still on study therapy or on study evaluations as of cutoff date. For patient who discontinued study therapy for reasons other than PD, radiological scans continued until a documented PD. After PD was documented, patient was considered off study. Patients were followed for survival status. In any study phase, patients could withdraw consent or become lost to follow-up 
A

Number at Risk

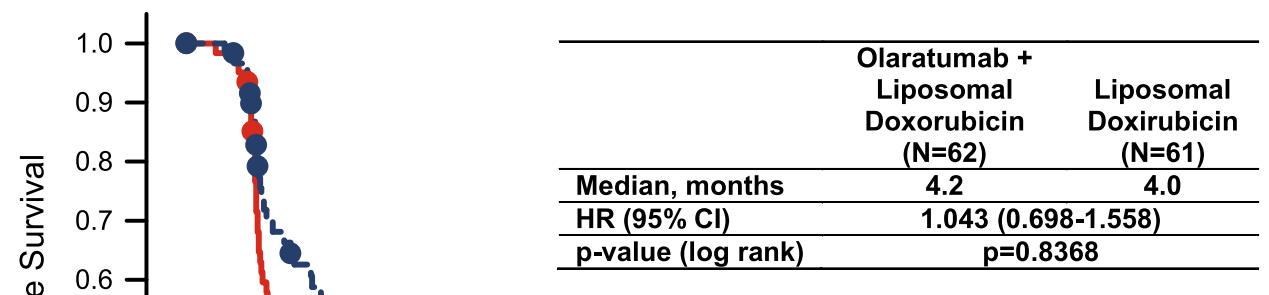

Olaratumab +

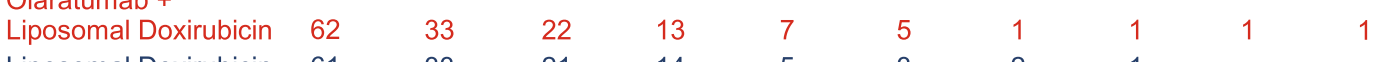

Liposomal Doxirubicin

6133

$21 \quad 14$

B

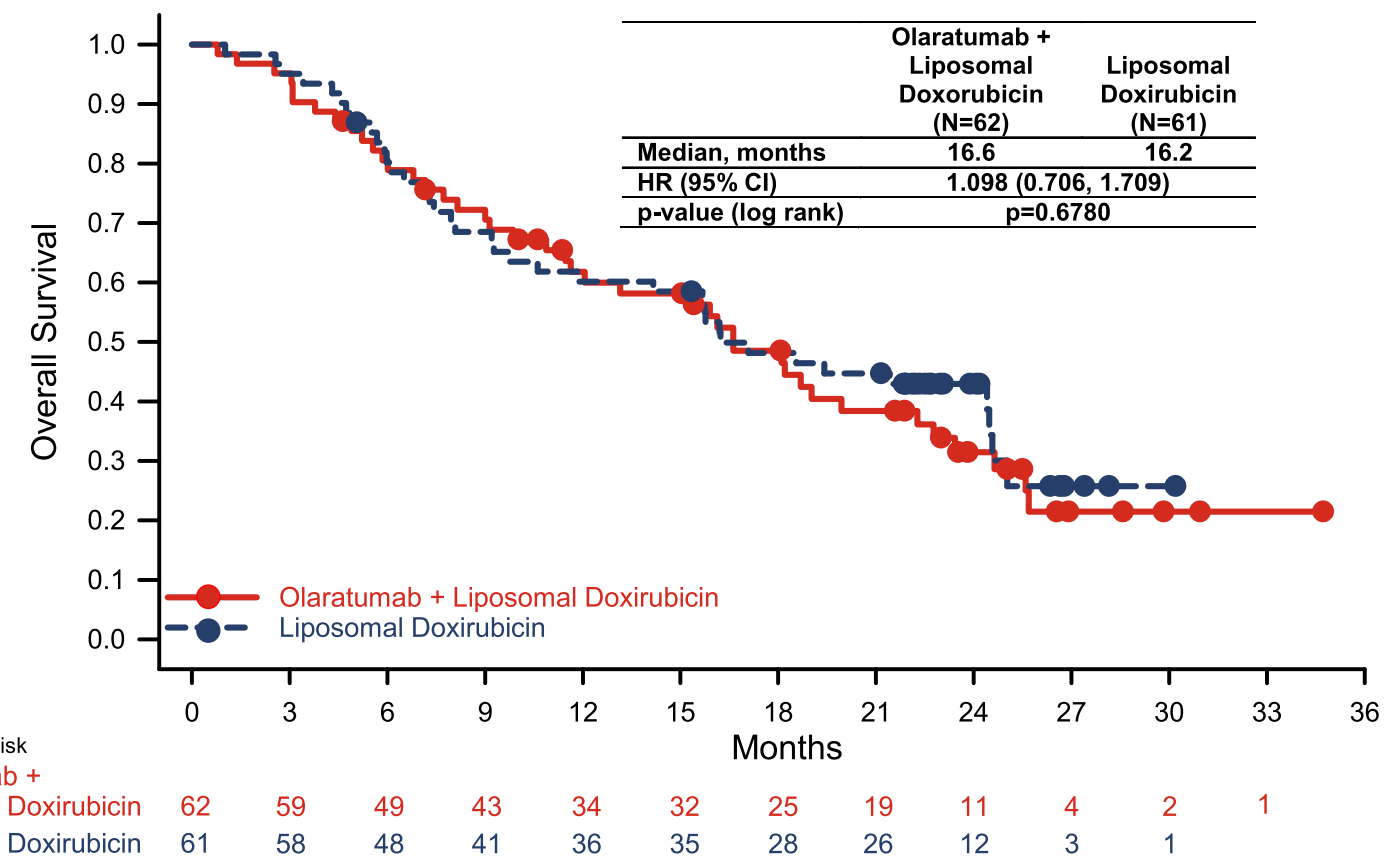

Fig. 2 Kaplan-Meier plots of progression-free (a) and overall (b) survival 


\section{Results}

\section{Demographics and disposition}

This study was conducted at 22 study sites in 3 countries (United States, United Kingdom, and Spain) between 11 June 2009 (first patient visit) and 13 February 2014 (last patient visit). The mITT population comprised 123 patients. Of these, the majority were White $(87.8 \%)$ (Table 1$)$. The median age was 59.0 years (range, $34-83$ years). The patients had an ECOG PS of either $0(55.3 \%)$ or 1 (44.7\%) at study entry. Overall, $75.6 \%$ of patients were platinum-resistant, whereas $24.4 \%$ were platinum-refractory. Following disease progression, 28 patients in the liposomal doxorubicin arm elected to receive olaratumab monotherapy.

Of 135 patients who entered the study, 125 were randomized and 123 were treated (62 olaratumab+liposomal doxorubicin, 61 liposomal doxorubicin) (Table 2). Two patients were randomized but not treated: One patient assigned to the olaratumab+liposomal doxorubicin arm discontinued for an unknown reason, and one patient assigned to the liposomal doxorubicin arm was not treated due to withdrawal by the patient. A total of 121 patients (61 in the olaratumab+liposomal doxorubicin arm, 60 in the liposomal doxorubicin arm) completed the study (Table 2). At the time of database lock, 2 patients were still on study therapy or on study evaluations. Fifty-four patients (43.9\%) discontinued study therapy because of progressive disease per RECIST, 18 patients (14.6\%) discontinued therapy because of symptomatic deterioration, and 2 patients $(1.6 \%)$ in the olaratumab+liposomal doxorubicin arm died. Both deaths occurred $\geq 21$ days after last dose of study treatment (27 and 21 days after the last olaratumab dose). One patient died due to progressive disease and the other due to pulmonary embolism considered by the investigator to be possibly related to study treatment. Nine patients (7.3\%) discontinued the study therapy due to AEs.

\section{Efficacy}

Forty-nine patients $(79.0 \%)$ in the olaratumab+liposomal doxorubicin arm and 47 patients (77.0\%) in the liposomal doxorubicin arm had a total of 96 PFS events. Median PFS was similar between groups (stratified $\mathrm{HR}=1.043 ; p=$ 0.837 ) (Fig. 2a). The 1-year PFS rate was $16.9 \%$ in the olaratumab+liposomal doxorubicin arm and $12.5 \%$ in the liposomal doxorubicin arm.

In the platinum-refractory subgroup, median PFS appeared slightly longer in the olaratumab+liposomal doxorubicin arm than in the liposomal doxorubicin arm (5.5 months vs 3.7 months $[\mathrm{HR}=0.85 ; 95 \%$ CI $0.38-1.91]$ ) (Table 3). In the platinum-resistant subgroup, median PFS was similar between groups (3.7 months in the olaratumab +liposomal doxorubicin arm vs 4.0 months in the liposomal doxorubicin arm; [HR =1.13; 95\% CI 0.71-1.80]) (Table 3).

Subgroup analysis showed that patients with disease duration of less than 15.2 months had improvement in PFS with olaratumab+liposomal doxorubicin treatment $(\mathrm{HR}=0.57$; 95\% CI $0.29-1.12)(n=50)$ compared with patients in the liposomal doxorubicin arm. Likewise, patients with a lower CA125 $(\leq 64.3)$ had higher PFS with olaratumab+liposomal doxorubicin treatment compared with patients in the liposomal doxorubicin arm, achieving an HR of 0.5 (95\% CI $0.21-1.22)(n=27)$. It should be noted that the $95 \%$ CIs for all considered subgroups covered a HR of 1.0, indicating no significant treatment difference on PFS between the 2 treatment arms.

For the secondary endpoints, a total of 44 OS events (censored) were observed across both study arms, including $21(33.9 \%)$ in the olaratumab+liposomal doxorubicin arm and $23(37.7 \%)$ in the liposomal doxorubicin arm. Median OS was similar between groups $(\mathrm{HR}=1.098$; 95\% CI $0.71-$ $1.71 ; p=0.678$ ) (Fig. 2b). The 1-year survival was $61.8 \%$ for patients receiving olaratumab+liposomal doxorubicin and $60.2 \%$ for patients treated with liposomal doxorubicin. The 2-year survival was 31.5 and $42.9 \%$ in the olaratumab+liposomal doxorubicin and liposomal doxorubicin arms, respectively. No statistically significant difference in OS was observed between the treatment groups.

With respect to overall tumor response, there were no complete responses (CRs) in either arm. The ORR (CR + partial response [PR]) was 12.9 and $16.4 \%$ in the olaratumab+liposomal doxorubicin and liposomal doxorubicin arms, respectively. The disease control rate $(C R+P R+$ stable disease) was $56.5 \%$ in the olaratumab+liposomal doxorubicin arm and $63.9 \%$ in the liposomal doxorubicin arm. Median duration of response was 39.1 weeks and 16.9 weeks in the olaratumab+liposomal doxorubicin and liposomal doxorubicin arms, respectively.

Table 3 Subgroup analysis of progression-free survival

\begin{tabular}{|c|c|c|c|c|c|c|c|c|c|c|}
\hline & \multicolumn{4}{|c|}{ Olaratumab + Liposomal Doxorubicin $(n=62)$} & \multicolumn{4}{|c|}{ Liposomal Doxorubicin $(n=61)$} & \multicolumn{2}{|c|}{ Hazard Ratio $^{a}$} \\
\hline & No. & Events & Median, months ${ }^{\mathrm{b}}$ & $95 \% \mathrm{Cl}^{\mathrm{b}}$ & No. & Events & Median, months ${ }^{\mathrm{b}}$ & $95 \% \mathrm{Cl}^{\mathrm{b}}$ & $\mathrm{HR}$ & $95 \% \mathrm{Cl}$ \\
\hline \multicolumn{11}{|c|}{ Stratification factor (from IVRS) } \\
\hline Platinum-refractory & 15 & 12 & 5.5 & $(1.6-9.2)$ & 16 & 13 & 3.7 & $(1.9-9.2)$ & 0.85 & $(0.38-1.91)$ \\
\hline Platinum-resistant & 47 & 37 & 3.7 & $(1.9-6.2)$ & 45 & 34 & 4.0 & $(2.7-7.8)$ & 1.13 & $(0.71-1.80)$ \\
\hline
\end{tabular}

$\mathrm{Cl}$, confidence interval; IVRS, interactive voice response system

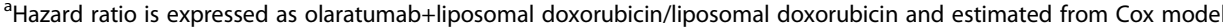

${ }^{b}$ Estimated by the Kaplan-Meier method 
Table 4 Treatment-emergent adverse events, regardless of causality

\begin{tabular}{|c|c|c|c|c|}
\hline & \multicolumn{4}{|l|}{ No. (\%) } \\
\hline & \multicolumn{2}{|c|}{ Olaratumab + Liposomal Doxorubicin $(n=62)$} & \multicolumn{2}{|c|}{ Liposomal Doxorubicin $(n=61)$} \\
\hline & All grades & Grade $\geq 3$ & All grades & Grade $\geq 3$ \\
\hline Patients with any TEAE & $62(100.0)$ & $37(59.7)$ & $61(100.0)$ & $40(65.6)$ \\
\hline \multicolumn{5}{|l|}{ Consolidated TEAE category ${ }^{a}$} \\
\hline Fatigue $^{\mathrm{b}}$ & $38(61.3)$ & $7(11.3)$ & $38(62.3)$ & $1(1.6)$ \\
\hline Mucositis ${ }^{c}$ & $30(48.4)$ & 0 & $28(45.9)$ & $4(6.6)$ \\
\hline $\operatorname{Rash}^{d}$ & $27(43.5)$ & $3(4.8)$ & $18(29.5)$ & $5(8.2)$ \\
\hline Abdominal pain ${ }^{e}$ & $24(38.7)$ & $2(3.2)$ & $30(49.2)$ & $8(13.1)$ \\
\hline Neutropenia ${ }^{f}$ & $20(32.3)$ & $8(12.9)$ & $13(21.3)$ & $5(8.2)$ \\
\hline Neuropathy ${ }^{9}$ & $12(19.4)$ & 0 & $9(14.8)$ & 0 \\
\hline Hypomagnesemiah $^{\text {h }}$ & $10(16.1)$ & 0 & $6(9.8)$ & $1(1.6)$ \\
\hline \multicolumn{5}{|l|}{ Preferred terms $s^{\mathrm{a}, \mathrm{i}}$} \\
\hline Nausea & $35(56.5)$ & $2(3.2)$ & $39(63.9)$ & $1(1.6)$ \\
\hline Constipation & $32(51.6)$ & $2(3.2)$ & $24(39.3)$ & 0 \\
\hline Vomiting & $21(33.9)$ & $3(4.8)$ & $20(32.8)$ & $6(9.8)$ \\
\hline Palmar-plantar erythrodysesthesia syndrome & $21(33.9)$ & $7(11.3)$ & $27(44.3)$ & $4(6.6)$ \\
\hline Diarrhea & 19 (30.6) & $2(3.2)$ & $13(21.3)$ & 0 \\
\hline Back pain & $16(25.8)$ & $1(1.6)$ & $10(16.4)$ & $1(1.6)$ \\
\hline Abdominal distension & $15(24.2)$ & $2(3.2)$ & $6(9.8)$ & $2(3.3)$ \\
\hline Urinary tract infection & $15(24.2)$ & 0 & $5(8.2)$ & $2(3.3)$ \\
\hline Headache & $12(19.4)$ & 0 & $7(11.5)$ & $1(1.6)$ \\
\hline Anemia & $10(16.1)$ & $3(4.8)$ & $13(21.3)$ & $1(1.6)$ \\
\hline Dysgeusia & $10(16.1)$ & 0 & $3(4.9)$ & 0 \\
\hline Dehydration & $9(14.5)$ & $3(4.8)$ & $3(4.9)$ & $2(3.3)$ \\
\hline Weight decreased & $8(12.8)$ & 0 & $4(6.6)$ & 0 \\
\hline Proteinuria & $7(11.3)$ & 0 & $2(3.3)$ & 0 \\
\hline Muscle spasms & $7(11.3)$ & 0 & $3(4.9)$ & 0 \\
\hline Pain in extremity & $4(6.5)$ & 0 & $9(14.8)$ & $1(1.6)$ \\
\hline \multicolumn{5}{|l|}{ TEAE of special interest } \\
\hline Infusion-related reactions ${ }^{j}$ & $6(9.7)$ & 0 & $3(4.9)$ & 0 \\
\hline Any SAE & $27(43.5)$ & $21(33.9)$ & $23(37.7)$ & $20(32.8)$ \\
\hline Discontinuation due to TEAE & $2(3.2)$ & n.r. & $7(11.5)$ & n.r. \\
\hline
\end{tabular}

AE, adverse event; MedDRA, Medical Dictionary for Regulatory Activities; n.r., not reported; SAE, serious adverse event; TEAE, treatment-emergent adverse event aTEAEs occurring in $\geq 10 \%$ of patients (all grades) and with $a \geq 5 \%$ between-arm difference (all grades or grade $\geq 3$ )

${ }^{\mathrm{b}}$ Consolidated term comprising the following synonymous MedDRA preferred terms: fatigue and asthenia

${ }^{C}$ Consolidated term comprising the following synonymous MedDRA preferred terms: aphthous stomatitis, mucosal inflammation, oropharyngeal pain, and stomatitis

${ }^{\mathrm{d} C}$ Consolidated term comprising the following synonymous MedDRA preferred terms: rash, rash follicular, rash generalized, rash macular, rash papular, rash pruritic, and rash pustular

e Consolidated term comprising the following synonymous MedDRA preferred terms: abdominal pain, abdominal pain lower, and abdominal pain upper ${ }^{\mathrm{f} C}$ Consolidated term comprising the following synonymous MedDRA preferred terms: leukopenia, neutropenia, neutrophil count decreased, and white blood cell count decreased

${ }^{9}$ Consolidated term comprising the following synonymous MedDRA preferred terms: hypoesthesia, neuropathy peripheral, paraesthesia, and peripheral sensory neuropathy

${ }^{h}$ Consolidated term comprising the following synonymous MedDRA preferred terms: blood magnesium decreased, hypomagnesemia, and magnesium deficiency 'Omits preferred terms that are included in consolidated categories

${ }^{j}$ Infusion-related reactions include a combination of specific preferred terms such as infusion-related reactions, anaphylaxis, and signs and symptoms such as flushing and itching 


\section{Safety}

Patients received a median of 4 cycles for each regimen (range 1, 24 for olaratumab+liposomal doxorubicin arm; range 1, 15 for liposomal doxorubicin arm). Of the 123 treated patients in this study, $41(66 \%)$ in the olaratumab+liposomal doxorubicin arm and 38 (62\%) in the liposomal doxorubicin arm had died at the time of data cutoff, disease progression being the most common cause of death (35 olaratumab+liposomal doxorubicin, 33 liposomal doxorubicin). Three patients $(4.8 \%)$ in the olaratumab+liposomal doxorubicin arm (1 SAE of disease progression, $1 \mathrm{SAE}$ of intracranial hemorrhage, and 1 SAE of pulmonary embolism) and 2 patients (3.3\%) in the liposomal doxorubicin arm (2 SAEs of disease progression) died due to an AE. Three deaths in each treatment arm were from other causes.

The rate of discontinuation due to AEs was higher in the liposomal doxorubicin arm (3.2\% vs $11.5 \%)$. The only reasons for discontinuation observed in more than 1 patient were pulmonary embolism and mucositis, both in patients in the liposomal doxorubicin arm. The incidence of AEs (all grades and grade $\geq 3$ ) was similar between the olaratumab+liposomal doxorubicin arm and the liposomal doxorubicin arm (all grades, $100 \%$ vs $100 \%$; grade $\geq 3,60 \%$ vs $66 \%$; any SAE, all grades, $43.5 \%$ vs $37.7 \%$ ). The most common treatment-emergent adverse events (TEAEs), regardless of causality (with $a \geq 5 \%$ between-arm difference), were fatigue- related (61\%), nausea (57\%), and constipation (52\%) with olaratumab+liposomal doxorubicin and nausea (64\%), fatigue-related (62\%), and mucositis (46\%) with liposomal doxorubicin (Table 4).

There were no cases of febrile neutropenia in either treatment arm. The rate of serious olaratumab-related infections in the olaratumab+liposomal doxorubicin arm was $1.6 \%$, as was the rate of liposomal doxorubicin-related infections in the liposomal doxorubicin arm.

\section{Discussion}

This study did not meet the primary endpoint of achieving a statistically significant improvement of PFS in the olaratumab+liposomal doxorubicin arm compared with liposomal doxorubicin alone in patients with advanced ovarian cancer. No statistically significant improvement in OS was achieved either, as a secondary endpoint of this study.

In general, safety profiles were similar between treatment arms, and AEs for olaratumab+liposomal doxorubicin were manageable and could be monitored easily. The higher incidence of neutropenia in the olaratumab+liposomal doxorubicin arm did not result in more febrile neutropenia.

There are limitations to this study. Although pretreatment tissue was collected in this study, the diagnostic antibody used to detect PDGFR $\alpha$ expression was subsequently found to have poor specificity for PDGFR $\alpha$ by also detecting PDGFR $ß$ [24], precluding any meaningful analysis of PDGFR $\alpha$ status of tissue samples. There were more discontinuations due to progressive disease according to RECIST criteria in the olaratumab+liposomal doxorubicin arm than in the liposomal doxorubicin arm. In addition to the possibility that this was a chance finding, this could reflect bias favoring patients in the investigational arm staying in this unblinded study until formal RECIST criteria for progression were met. This might also explain why discontinuation for toxicity occurred more frequently in the liposomal doxorubicin arm. Nonetheless, most of the AEs observed in this study are consistent with the known safety profile of liposomal doxorubicin or occur in the metastatic ovarian cancer population.

\section{Conclusions}

There was no statistically significant difference between the olaratumab+liposomal doxorubicin arm and the liposomal doxorubicin arm in PFS or secondary endpoints of OS and ORR. Olaratumab given in combination with liposomal doxorubicin was well tolerated.

\section{Abbreviations}

AE: Adverse event; CA125: Cancer antigen 125; Cl: Confidence interval; CR: Complete response; ECOG PS: Eastern Cooperative Oncology Group performance status; EOC: Epithelial ovarian cancer; HR: Hazard ratio; IgG1: Immunoglobulin G subclass 1; IV: Intravenous; IVRS: Interactive Voice Response System; IWRS: Interactive Web Response System; MAPK: Mitogenactivated protein kinase; mITT: Modified intent-to-treat; NCI-CTCAE: National Cancer Institute Common Terminology Criteria for Adverse Events;

ORR: Objective response rate; OS: Overall survival; PD: Progressive disease; PDGF: Platelet-derived growth factor; PDGFR: Platelet-derived growth factor receptor; PFS: Progression-free survival; PR: Partial response; RECIST: Response Evaluation Criteria in Solid Tumors; SAE: Serious adverse event; SRC: Safety Review Committee

\section{Acknowledgements}

Robert Panek, PhD and Lori Kornberg, PhD (both of Syneos Health, Raleigh, NC), assisted with the writing of this article.

\section{Funding}

This work was sponsored by ImClone System LLC, a wholly owned subsidiary of Eli Lilly and Company.

The study was designed by the funder, ImClone /Eli Lilly and Company, with input from ovarian cancer experts. The data were analyzed and interpreted by ImClone /Eli Lilly and Company in collaboration with the academic authors. All authors had access to all of the data and vouch for the accuracy and completeness of the data and analyses reported and for the fidelity of the study to the study protocol. All authors had final responsibility for the decision to submit for publication. All authors participated in the drafting of the manuscript and/or critical revisions of subsequent drafts. Writing and editorial assistance was provided by Syneos Health on behalf of ImClone /Eli Lilly and Company.

\section{Availability of data and materials}

Lilly provides access to the individual patient data from studies on approved medicines and indications as defined by the sponsor specific information on ClinicalStudyDataRequest.com. This access is provided in a timely fashion after the primary publication is accepted. Researchers need to have an approved research proposal submitted through

ClinicalStudyDataRequest.com. Access to the data will be provided in a secure data sharing environment after signing a data sharing agreement. 


\section{Authors' contributions}

WPM, RTP, MG, and ACH were involved in data acquisition and data analysis/ interpretation. WPM was also involved in conception and design of the work. $\mathrm{PP}, \mathrm{AS}, \mathrm{RI}$ were involved in data analysis/interpretation. All authors reviewed the manuscript and contributed to writing and/or critical revision of the manuscript. All authors read and approved the final version for submission.

\section{Ethics approval and consent to participate}

The study (NCT00913835) was conducted in accordance with the International Conference on Harmonisation Good Clinical Practice guidelines, the Declaration of Helsinki, and local laws and regulations. The study was approved by the following ethical review boards: Medstar Research Institute-Georgetown University Oncology Institutional Review Board; King's Hospital Research Ethics Committee-King's College Hospital; South East London Rec 3-King's College Hospital; London-Dulwich Charing Cross Hospital; Henry Ford Health System. Institutional Review Board; Indiana University-Purdue University Institutional Review Board; Dana Farber Cancer Institute Office for Human Research Studies; Dana Farber Cancer Institute Office for the Protection of Research Subjects; University of California-Irvine IRB; Chesapeake Research Review; Presbyterian Healthcare System Institutional Review Board; Hospital Clinico San Carlos, Servicio Farmacologia Clinica 1a planta Ala Norte-Ciudad Universitaria; Atlantic Health System, Institutional Review Board; University of Southern California IRB. All patients provided written informed consent.

\section{Consent for publication}

Not applicable.

\section{Competing interests}

WPM, MG, and ACH report no conflicts related to this manuscript. RTP reports research funding and $S A B s$ within de minimis.

PP, AS are employees of Eli Lilly and Company and own stock. RI was an employee of Eli Lilly and Company at the time of manuscript preparation. He is currently employed by Celgene Corporation.

\section{Publisher's Note}

Springer Nature remains neutral with regard to jurisdictional claims in published maps and institutional affiliations.

\section{Author details \\ ${ }^{1}$ Virginia Commonwealth University, 1201 E Marshall St, Room 11-210, Richmond, VA 23298, USA. ²Massachusetts General Hospital, Yawkey 9-064, 32 Fruit St, Boston, MA 02114, USA. ${ }^{3}$ The Royal Marsden Hospital, Fulham Road, London SW3 6JJ, UK. ${ }^{4}$ Hospital Clinico, San Carlos Servicio de Oncologia Medica, Madrid, Spain. ${ }^{5}$ Eli Lilly and Company, Indianapolis, IN, USA. ${ }^{6}$ Eli Lilly and Company, Lilly UK, EMC Building, Erl Wood Manor, Windlesham, Surrey GU20 6PH, UK. ${ }^{7}$ Celgene Corporation, 86 Morris Ave, Summit, NJ 07901, USA.}

Received: 27 June 2018 Accepted: 9 December 2018

Published online: 27 December 2018

\section{References}

1. American Cancer Society. Cancer Facts \& Figures 2018. In: Atlanta: American Cancer Society; 2018.

2. Treatment of invasive epithelial ovarian cancers, by stage. American Cancer Society, Atlanta. 2017. https://www.cancer.org/cancer/ovarian-cancer/ treating/by-stage.html. Accessed 15 Jan 2018.

3. Ledermann JA, Raja FA, Fotopoulou C, Gonzalez-Martin A, Colombo N, Sessa C. Newly diagnosed and relapsed epithelial ovarian carcinoma: ESMO clinical practice guidelines for diagnosis, treatment and follow-up. Ann Oncol. 2013;24:vi24-32.

4. NCCN Clinical Practice Guidelines in Oncology: Ovarian Cancer. Version 1. 2018. National Comprehensive Cancer Network, Ft. Washington, PA. 2018. Accessed 20 Apr 2018

5. Markman M, Hoskins W. Responses to salvage chemotherapy in ovarian cancer: a critical need for precise definitions of the treated population. J Clin Oncol. 1992;10:513-4.

6. Verhaar-Langereis M, Karakus A, van Eijkeren M, Voest E, Witteveen E. Phase II study of the combination of pegylated liposomal doxorubicin and topotecan in platinum-resistant ovarian cancer. Int J Gynecol Cancer. 2006; 16:65-70.
7. Salom E, Mirhashemi R, Lambrou N, Almeida Z, Auguste F. Topotecan and doxorubicin $\mathrm{HCL}$ liposome combination therapy in the treatment of recurrent/refractory ovarian cancer: a phase II study. Proc am Soc Clin Oncol. 2003;22:1911.

8. Horwood K, Colosimo M, Wyld D, Abraham R, Choo PS. Crandon a. a phase II trial of gemcitabine and liposomal doxorubicin in platinum-resistant ovarian cancer (PROC). Proc Am Soc Clin Oncol. 2002;21:888.

9. D’Agostino G, Ferrandina G, Ludovisi M, Testa A, Lorusso D, Gbaguidi N, et al. Phase II study of liposomal doxorubicin and gemcitabine in the salvage treatment of ovarian cancer. Br J Cancer. 2003;89:1180-4.

10. Campos SM, Matulonis UA, Penson RT, Lee H, Berkowitz RS, Duska LR, et al. Phase II study of liposomal doxorubicin and weekly paclitaxel for recurrent Müllerian tumors. Gynecol Oncol. 2003;90:610-8.

11. Gallego O, Monetsinos J, Losa F, Eres N, Arcusa A, Franquesa R. a phase II study of pegylated liposomal doxorubicin (PLD), and cyclophosphamide (CTX) as second line therapy in platinum resistant ovarian cancer (OC) patients. Proc am Soc Clin Oncol. 2003;22:1931.

12. Katsaros D, Oletti MV, Rigault de la Longrais IA, Ferrero A, Celano A, Fracchioli S, et al. Clinical and pharmacokinetic phase II study of pegylated liposomal doxorubicin and vinorelbine in heavily pretreated recurrent ovarian carcinoma. Ann Oncol. 2005;16:300-6.

13. European Medicines Agency. Caelyx. https://www.ema.europa.eu/medicines/ human/EPAR/caelyx\#product-information-section. Accessed 3 Oct 2018.

14. Food US, Adminstration D. Drugs@FDA: FDA approved drug products. DOXIL (LIPOSOMAL). https://www.accessdata.fda.gov/drugsatfda_docs/label/ 2016/050718s051 lbl.pdf. Accessed 3 Oct 2018.

15. Hart CE, Forstrom JW, Kelly JD, Seifert RA, Smith RA, Ross R, et al. Two classes of PDGF receptor recognize different isoforms of PDGF. Science. 1988;240:1529-31.

16. Heldin $\mathrm{CH}$, Ostman A, Ronnstrand L. Signal transduction via platelet-derived growth factor receptors. Biochim Biophys Acta. 1998;1378:F79-F113.

17. Ostman A, Heldin CH. Involvement of platelet-derived growth factor in disease: development of specific antagonists. Adv Cancer Res. 2001;80: $1-38$.

18. Ng F, Boucher S, Koh S, Sastry KS, Chase L, Lakshmipathy U, et al. PDGF, TGFbeta, and FGF signaling is important for differentiation and growth of mesenchymal stem cells (MSCs): transcriptional profiling can identify markers and signaling pathways important in differentiation of MSCs into adipogenic, chondrogenic, and osteogenic lineages. Blood. 2008;112:295-307.

19. Chen CY, Liu SH, Chen CY, Chen PC, Chen CP. Human placenta-derived multipotent mesenchymal stromal cells involved in placental angiogenesis via the PDGF-BB and STAT3 pathways. Biol Reprod. 2015;93:103.

20. Choi H-J, Armaiz Pena GN, Pradeep S, Cho MS, Coleman RL, Sood AK. Antivascular therapies in ovarian cancer: moving beyond anti-VEGF approaches. Cancer Metastasis Rev. 2015;34(1):19-40.

21. Lassus $H$, Sihto $H$, Leminen A, Nordling $S$, Joensuu H, Nupponen NN, et al. Genetic alterations and protein expression of KIT and PDGFRA in serous ovarian carcinoma. Br J Cancer. 2004;91:2048-55.

22. Wilczynski SP, Chen YY, Chen W, Howell SB, Shively JE, Alberts DS. Expression and mutational analysis of tyrosine kinase receptors c-kit, PDGFRalpha, and PDGFRbeta in ovarian cancers. Hum Pathol. 2005;36:242-9.

23. Matei D, Emerson RE, Lai YC, Baldridge LA, Rao J, Yiannoutsos C, et al. Autocrine activation of PDGFRalpha promotes the progression of ovarian cancer. Oncogene. 2006;25:2060-9.

24. Holzer TR, O'Neill Reising L, Credille KM, Schade AE, Oakley GJ. Variability in platelet-derived growth factor receptor alpha antibody specificity may impact clinical utility of immunohistochemistry assays. J Histochem Cytochem. 2016;64(12):785-810.

25. Matsuo K, Nishimura M, Komurov K, Shahzad M, Ali-Fehmi R, Roh JW, et al. Platelet-derived growth factor receptor alpha (PDGFRa) targeting and relevant biomarkers in ovarian carcinoma. Gynecol Oncol. 2014;132:166-75.

26. Matei $D$, Emerson RE, Schilder J, Menning N, Baldridge LA, Johnson CS, et al. Imatinib mesylate in combination with docetaxel for the treatment of patients with advanced, platinum-resistant ovarian cancer and primary peritoneal carcinomatosis: a Hoosier oncology group trial. Cancer. 2008;113:723-32.

27. Loizos N, Xu Y, Huber J, Liu M, Lu D, Finnerty B, et al. Targeting the platelet-derived growth factor receptor alpha with a neutralizing human monoclonal antibody inhibits the growth of tumor xenografts: implications as a potential therapeutic target. Mol Cancer Ther. 2005;4: 369-79. 
28. Lowery CD, Blosser W, Dowless M, Knoche S, Stephens J, Li H, et al. Olaratumab exerts antitumor activity in preclinical models of pediatric bone and soft tissue tumors through inhibition of platelet-derived growth factor receptor a. Clin Cancer Res. 2018:24:847-57.

29. Tap WD, Jones RL, Van Tine BA, Chmielowski B, Elias AD, Adkins D, et al. Olaratumab and doxorubicin versus doxorubicin alone for treatment of softtissue sarcoma: an open-label phase $1 \mathrm{~b}$ and randomised phase 2 trial. Lancet. 2016;388:488-97.

Ready to submit your research? Choose BMC and benefit from:

- fast, convenient online submission

- thorough peer review by experienced researchers in your field

- rapid publication on acceptance

- support for research data, including large and complex data types

- gold Open Access which fosters wider collaboration and increased citations

- maximum visibility for your research: over $100 \mathrm{M}$ website views per year

At $\mathrm{BMC}$, research is always in progress.

Learn more biomedcentral.com/submissions 